Hespital de Niñes Manuel Arriaran

Seciòa Medicina Interna.

\title{
NEFROSIS EN LA INFANCIA $(*)$
}

\author{
por c) Dr. ARTURO BAEZA GONI
}

\section{Introducciōn.}

El objeto de este trabajo, que por mandato de los organizadores del Primer Congreso Pan Americano de Pediatria, presentamos en calidad de co-relato junto con México, no tiene otro alcance que exponer la experiencia chilena sobre una materia que está en el tapete de la discusión. desde que Müllcr, en 1905. desglosara del Mal de Bright, las afecciones degenerativas del riñón, dándoles el nombre de nefrosis para distinguirlas de las nefritis, en que el elemento inflamatorio glomerular seria el determinante.

Carece nuestra ponencia de originalidad, desde luego. pues no han estado a nuestro alcance ni preparación. los medios suficientes para ralizar sobre la materia un estudio de invertigación pura, digno de ser expuesto en un torneo de esta naturaleza. Por otra parte. preocupados desde hace tiempo en forma casi exclusiva de las nefropatias agudas del niño, debemos confesar que el estudio de las nefrosis lo habíamos dejado. dada su complejidad e importancia. para una época posterior, en que pudiéramos consagrarle una mayor dedicación.

Nos limitaremos. pues, previo un somero resumen de la abundantísima literatura, conocida por demás de Uds., a exponer lo más brevemente posible. la experiencia recogida a través de una revisión de la casuística observada en nuestros tres centros de atención infantil de la capital. los Hospitales Manucl Arriarán. Roberto del Río y Calvo Mackenna.

(*) Trabajo presentado al primer Congreso Panamericano de Pediatria, réebrade on Washington, en julio de 1947. 
Conceptos generales sobre nefrosis.

Dentro de la denominación de nefrosis comprendemos aquellos procesos mórbidos, que. con caracteres clínicos más o menos bien definidos, tienen como substrato anatómico lesiones degenerativas del parénquima renal.

Desde el punto de vista de su evolución clínica, podemos distinguir dos grupos más o menos bien individualizados: las nefrosis agudas y las crónicas (Jiménez Diaz).

Las nefrosis agudas suelen presentar como único síntoma una albuminuria más o menos apreciable. Son frecuentes en 1 curso de algunas afecciones febriles (nefrosis albuminúrica febril) y se caracterizan en general por su benignidad, curando tan pronto como lo hace su afección causal (neumonia, tifoidea, difteria, etc.). Otras veces revisten caracteres graves y a la albuminuria se agregan edemas de cierta intensidad y síntomas generales de mayor importancia; en otros casos su evolución es rápidamente fatal y sin que durante la vida se observen sintomas propiamente renales, en la autopsia nos encontramos con extensas lesiones necróticas del parénquima renal.

Una clasificación anátomo-patológica $\mathrm{d}_{e}$ las diversas formas de nefrosis, agudas o crónicas, leves, de mediana in ${ }^{-}$ tensidad o muy graves, no es fácil, por cuanto teniendo muchas veces esta afección tendencia a la mejoría, los estudios histopatológicos al respecto no son abundantes, asi que es difícil establecer etapas bien definidas en la evolución de sus lesiones.

Los estudios más prolijos a este respecto, son los de Fahr, qui $\in$ n sobre material fresco pudo distinguir tres grados principales: 1) Degeneración albuminosa caracterizada por un simple enturbiamiento del protoplasma de los epitelios tubulares. Proceso reversible y que explicaría las formas albuminúricas leves. 2) Degeneración gutural hialina, con destrucción de parte de los epitelios tubulares, que $\in n$ un grado más avanzado puede llegar a la degeneración e infiltración grasa (lipoidosis). A este grado corresponderian algunas formas agudas $y$ otras que evolucionan a la cronicidad. pudiendo todavía reparatse o curar. 3) Nefrosis epitelial, con destrucción intensa de gran número de células epiteliales y que se acompaña de depósito de sales calcáreas. Sería ésta la nefrosis necrotizante mortal. 
Desde el punto de vista de su etiología, las nefrosis, tanto de evolución aguda o crónica, ya sean éstas leves, de mediana intensidad o mortales, pueden obedecer a diversas causas, a saber: 1) Agentes quimiotóxicos, como el Hg. sales de cro, arsenicales, bismuto, etc., que producen generalmente las formas más graves de tipo necrótico. 2) Procesos infecciosos o bacterianos agudos, como la tifoidea, difteria, neumonía, influenza, toxicosis, cólera, o bien crónicos, como sífilis, tuberculosis, malaria, trastornos nutritivos crónicos, los cuales pueden revestir toda la gama evolutiva ya siñalada. 3) Trastornos metabólicos, como la diabetes, obesidad. Basedow. 4) El grupo de las nefrosis sin causa apreciable y que evolucionan como tales exclusivamente o con mucha mayor frecuencia en relación indiscutible con un proceso glomérulo-nefrítico, sin que entre sus antecedent $\ll s$ prolijamente tomados, encentremos su etiología.

Entre las formas agudas, nosotros hemos observado numercses casos de albuminurias febriles, en el curso de algunas afecciones agudas, principalmente neumonías a tifoideas. En las primeras, siempre de evolución benigna y que han curado junto con el proceso causal. En la tifódea, en cambio. hemos visto formas graves que han presentado edema, albuminuria más o menos acentuada e hipoproteinemia y que han mejorado después de gravis alternativas. En un caso que llegó a la muerte, se encontró en la autopsia una necrosis intensa del parénquima renal, sin que durante la evolución de la enfermedad se observatan sintomas renales.

Revisando protocolos de autopsias. pudimos reunir un conjunto de 27 casos de nínos fallecidos por diferentes causas: en los cuales se encontró. como hallazgo anatómico, una lipoidosis acentuada de los tubulis, sin alteraciones inflamatorias glomerulares.

Séralamos estos hechos por parecerncs de interés para el futuro, pues una mejor investigación nos ayudará a precisar, con mayor acuciosidad, los caracteres clínicos que deben concurrir en estos enfermitos.

Respecto a las formas crónicas más frecuentes en cl niño pequeño que en el adulto, diremos que este concepto de cronicidad, si bien es una característica resaltante en muchos casos, no lo es siempre, por cuanto hay una considerable variedad de tipos en su evolución. Asi hay casos en que la afección, con las caracteristicas que señalaremos más adelan- 
te, evoluciona en días, semanás o meses, a veces con extraordinaria intensidad, otras benignas y de evolución breve.

Siendo las llamadas nefrosis crónicas o sindromas nefrósicas, entidades clínicas bien definidas, que por sus características tan especiales y problemas en pleno estudio, merecen un estudio especial, trataremos de realizarlo en la forma más completa posible.

Constituyen ellas, sin duda, el capítulo más interesante de este estudio y que ha merecido la nayor preacupación de investigadores y de clinicos, disde que Münk, en 1913. Volbard y Fabr, Epstein, Loblein. Sternberg y tantos otros le señalaran a esta afección una individualidad tan caracteristica. Se ha abierto desde entonces una discusión que está aún muy lejos de ser agotada, permaneciendo aún muchos puntos oscuros de gran intetés y a cuya solución no hacemos sino aportar un leve esfuerzo, con nuestra modesta contribución y estamos ansicsos de oír en esta reunión nuevos. conceptos. que nos han de servir para aprevechat mejor en ef futuro el material que de aquí en adelante se nos presente.

Se define como nefrosis crónica lipoidea aquel proceso que clinicamente $s e$ caracteriza por una sintomatología bien definida. Se trata de afecciones más frecuentes en el niño pequeño que en el adulto, de comienzo más o menos insidiose, sin relación.demostrable claramente con un procesc causal, cayas manifestaciones clínicas fundamentales son: albuminuria acentuada. edema que a veces alcanza proporciones $d_{\varepsilon}$ anasarca, hipoproteinemia con cucciente proteics bajo, hipercelesterinemia. lipoiduria con presencia de cristales birrefringentes, metabolismo basal gencralmente disminuido. anemia macrocítica casi constante, leucocitosis con marcada desviación a la izquierda. Sedimentación alta. El funcionamiento renal no está alterado, no existe hipertensión ni tampoco hematuria. Su evolución puede ser de semanas, meses. y aún años, terminando a veces con la muerte, y en algunas ocasicnes curando en forma ispectacular.

Desde el punto de vista de su anatomia patológica, ésta se caracteriza por lesiones degenerativas importantes en of epitelio tubular; algunes rúbulos están dilatados y llenos de células aplanadas, mientras otros se presentan con su lumen estrechado, debido a la gran hinchazón de las células de sus epitelios. Existe notable depósito de grasas y lipoides (colesterol) en las células, dando a la luz polarizada el aspecto birrefringente. En los glomérulos se observan manifestacio- 
nes de tipo degenerativo hjalino, con depósito también de grasa, pero sin existir proliferación de sus células endoteliales, ni alteraciones inflamatorias de ninguna especie.

Concebido así el cuadro de esta afección, parecería lógico otorgarle una individualidad nosológica perfectamentr definida, Así, en realidad. lo sostuvieron los primeros investigadores, quienes la denominaron nefrosis lipoidea genuina, a la cual Epstein. fundado en hechos por él comprobados. pretendió separarla del grupo de las nefropatías propiamente tales, considerándola como una afección del metabolismo. principalmente de las albúminas $y$ lipoides, comparable a una verdadera diabetes albuminúrica.

Sin embargo, estudios posteriores que comprobaron la rareza con que se observa en su forma pura o genuina, la relativa frecuencia con que se presenta, con caracteres idénticos, en el curso de ciertas glomérulo-nefritis, y el hallazgo casi constante (para algunos autores siempre existirian) de alteraciones inflamatotias glomerulares $\mathrm{en}$ la mayor parte de los casos descritos como tales, ha llevado a sostener a un grupo, respetable también, de investigadores, que dicho cuadro no existe como entidad clínica primaria, sino que se trata de procesos degenerativos del parénquima renal. secundarios a una lesión primitiva glomerular de carácter inflamatorio. Resultando todo el proceso no de una alteración del metabolismo proteico, sino que la alteración glomerular produciría, por una parte, una perturbación nutritiva del epitelio tubular, que se nutre a través de los vasos eferentes del glomérulo, y por otra, dicho glomérulo alterado se haría permeable para las albúminas, las cuales, al ser eliminadas a través de los túbulos, contribuirian a favorecer la degeneración de sus epitelios ya lesionados en su nutrición.

Ambas teorias son defendidas por sus partidarios con argumentos cada vez más sólides, que, sin embargo, son posteriormente refutados por nuevos hallazgos. frutos de concienzudas y pacientes investigaciones.

Llenariamos páginas y páginas si quisiéramos exponer y analizar todo el material acumulado al respecto. Como no es éste el objeto de nuestra ponencia, nos limitamos a recomendar la lectura de trabajos tan documentados como los de Fishberg, Hadfield and Garrod, Jiménez Díaz, Enrique Varela, Holt and Mc Intosh. Griffith Mitchell, Heymann and Startzman, Aldrich, etc., quienes han agotado, puede decirse, los estudios más modernos al respecto. 
De su atenta lectura $y$ revisión, asociada a nuestra experiencia personal, podemos deducir que el estado actual de tan debatido asunto, podria sintetizarse en los siguientes conceptos:

1. Si bien la nefrosis lipoídea genuina se encuentra con una rareza extracrdinaria (Leiter: 1 a $5 \%$ de las nefropatias), no puede todavía set negada su existencia, $y_{\text {id }}$ qus existen numeroses casos, descritos por autores de recorocida autoridad. que reúnen todos los atributos señalados para aceptarlos como tal (Holt. Howland, Marriot, Clausen. Scwartz, Hitzcot, Head, Murphy, Warfield, Heymann). Cemo lo señala el mismo Jiménez Díaz, uno de los contradictores de esta teoría. "aunque rara, no es imposible su existencia".

2. No parece demostrada la teoria de Epstein del origen metabólico primitivo de la afección, aun cuando en ella se observe, en algunos casos al menos, una relación evidente, perc todavía inexplicada, con factores constitucionales o endocrinos. principalmente tiroídecs. hepáticos, hipofisiatics, etc., $y$ algunos autores hayan creído demostrar la existencia de proteínas descaracterizadas (Goetsch y Sytle), que al ser eliminadas por al riñón, preducirian la degeneración tubular secundaria.

3. Exicte acuerdo, al parecer unánime, en estimar que en la gran mayoria de los casos, la afección es de origen renal primario. y que la alteración degenerativa del epitelio tubular secundaria a un proceso glomerular, explica la patogenia de todos sus síntomas. Sin embargo, aun no está completamente demostrado cuál es el proceso patológico primitivo del glomérulo, punto de partida para la mayoria de los autores, de todo el cuadro, y aun cuando diversas experiencias (Meclean, Fishberg, Vorzimmer) han demostrado una permeabilidad anormal para las albúmínas en estos casos. sin embargo. la fisio-patología aun no explica el mecanismo de este fenómeno de tanta importancia.

4. Estimamos, por lo tanto, que en el momento actual debemos distinguir dos tipos de entidades clinicas $y$ anátomo-patológicas: la nefrosis genuina y lo que debe denominarse sindroma nefrósico secundario a procesos primitivos del glomérulo (Nephrosis-Nephritis de Gray), de carácter inflamatorio típico, o bien, cuya patogenia está aủn 
por dilucidar, para lo cual un aporte de primera categoría nos parece el resultado de las recientes experiencias de Trueta (Oxford), quien parece haber demostrado que por un mecanismo neurovascular se pueden provocar altaraciones glomerulares, sin que sea necesaria la acción de un proceso inflamatcrio o alérgico de estos órganos. Seguramente el porvenir nos señalará si estos hechos tienen la verdadera importancia que hoy por bey tiende a dársele.

Si la patogenia de la nefrosis crónica presenta puntos oscuros, difíciles de dilucidar por ahora, sucede lo mismo con lo que respecta a su etiología. En general se acepta que todos estos procesos tienen un origen infeccioso. Así, Linneweh. de la Clínica de Bessau en la Charité, sostiene, basado en algunas experiencias personales, que se trata de infecciones atenuadas, principalmente estreptocócicas o neumocócicas, que en forma lenta lesionarian al epitelio renal, desencadenando el cuadro después de una alteración primitiva del glomérulo. Diversas afecciones han sido culpadas también de ser la causa de este proceso tan singular y así no hay qua olvidar que los primeros casos descritos como tales, por Munk, reconocían al parecer como causa la sifilis. También la concomitancia tan frecuente de la nefrosis con la erisipela y scbre todo con las neumococias, hizo pensar a muchos investigadores que algunos de ellos fuera el germen tiológico. aun cuando hoy día se acepta que se trata más bien d: una disminución de la inmunidad en un organismo edematoso y que estos gérmenes aprovecharían, para producir una peritonitis, una sepsis neumocócica o una erisipela.

Es indiscutible, por lo demás, de que, si bien es cierto, algunos casos de nefrosis o $\mathrm{d}_{2}$ sindrome nefrótico, parecen obedecer a una causa determinada. que radica en procesos infecciosos, principalmente amigdalianos o sobre todo de la piel, como sucede entre nosotros y que ciertos y determinades casos han curado al extirpar oportunamente un foco, dentario v. gr., no es menos cierto que, por lo menos, en la mitad de los casos. aunque se busque prolijamente la causa. ésa pirmanece desconocida.

Para terminar esta ya larga exposición sobre las generalidades de este tema. debemos consid rar lo que se refiere al tratamiento y al pronóstico de esta enfermedad.

Tanto en el tratamiento de la nefrosis genuina como en el sindrome nefrósico. los grandes progresos que se esperaba obterier después de las investigaciones de Epstein y colabora- 
dores, sobre su patogenia, sólo en parte se han realizado, pues. a pesar de1 uso de preparados tiró́deos y del reemplazo de las albuminas perdidas por su eliminación constante en la orina. per un régimen hiperproteico, el cuadro siempre se caracteriza por su rabeldia a toda clase de tratamientos empleados, y si bien un número considerable de casos mejoran. lo hacen después de meses o años de tórpida evolución: muchas vieces en forma inexplicable. El edema suele ser rebelde aún al uso de los diuréticos mercuriales. los cuales presentan a su haber algunos fracasos mortales. Lu urea, tan ampliamente recomendada por algunos autores. ftacasa lamentablemente en manos de otres, algo semejante podemos decir de la plasmoterapia que nos hizc concebir justificadas esperanzas, en igual forma que el uso de aminoácidos, de preparados tiroídeos, etc.

En general, el tratamiento debe sir sintomatico y las medidas a tomar deben estar dirigidas sobre todo a conserva: en las mejores condicionss al paciente, dándole tiempo al organismo para que se establezcan en él las condiciones. aun completamente desconocidas, en que la curación ha de producirse a veces en forma inesperada. En este sentido, sin duda. lo más importants será evitar y combatir enérgicamente todo proceso infeccioso, ya sea originario o intercurrente. Creemos que las mejorías que hoy día se observan con mucha maycr frecuencia que antes, se deben, por sobre todo, al uso oportuno de la sulfa y penicilinoterapia. y a un tratamient? higiénico dietético más racional.

No hemos comprendido en este trabajo da nefrosis amiboídea. por creerla de menos interés, pues. aun cuando la hemos observado en el curso de algunos procesos supurativos crónicos, principalmente de origen tuberculoso, no creemos del caso exponerlas en detalles: su estudio no ofrece. por to demás, particularidades en la infancia que la distingan d: las formas del adulto.

\section{Resultado de nuestras observaciones clínicas.}

Desde 1930 hasta la ficha, han pasado por los tres principales servicios de atención infantil de la capital. centenares de niños afectos de nefropatias de diversa índole. La gran mayoría de ellos présentaron el tipo de la glomérulonefritis difusa, enfermedad extraordinariamente frecuente en nuestro medio. El fruto de un análisis minucioso, realizado par nosotros, sobre este abundante materiat. aparece publica- 
do en la monografia titulada "La glomérulo-nefritis en la infancia" (Editorial Zig-Zag, 1942), en la cual, después de analizar los diversos aspectos de la enfermedad, relatamos 156 observaciones de esta afección. controladas personalmente. Posteriormente hemos revisado más de 400 nuevos casos. propios y de otros autores, de tal manera que nuestra experiencia clínica sobre la materia, representa un acúmulo de más de 500 enfermos. los cuales hemos estudiado de acurtdo con las posibilidades y recursos a nuestro alcance.

En la sección Temas Libres haramos una exposición detallada sobre algunas particularidades especiales encontradas por nosotros.

Respecto a nefrosis crónica nos ha llamado siempre la atención la rareza de su frecuencia. considerada como entidad pura o genuina. al lado de la relativa frecuencia con que observamos el sindroma ncfrótico, como acompañante de algunas formas de glomérulo-nefritis.

En 1930 publicamos por primera vez, en conjunto con los Dres. Alfredo Oyarzún Lorca y Ernestina Peña, un caso de Nefrosis Lipoídea, cuya evolución clínica parecía reunir todas las características para considerarla como tal.

Enfermo de 12 años de adad. sill etjolog a pricisa, conienzo insidioso anasaria. presiones normales durante toda sa avolución, albuminuria hasta cifta: de $50 \mathrm{grs}$., hipoproteinemia de $3.5 \mathrm{grs}$. con en xiente proceico destendido, exlesterinemia de 2.51 grs.. metabolismo dismin'uido in $70 \%$. bematuria muy escasa e inconstante. Fatuece for una netmonia stguida de peritonitis neumocó cica. Sin embargo. la auropsia practicada por ta Dra. Enestina Feña reveló. a) lado de has lesiones aractaricticas de la nefrosis lipoiclea. lesiones visibles de g’omérula-nefritis.

Esta observación adolece. desgraciadamise, de ta falta de un estudio his. topatológico completo.

Bauzá y Hernández, en el Hospital Roberto del Río. realizan un estudio sobre 27 casos de nefrosis reunidos en el lapso de 12 años y después de un detenido análisis, llegan a la conclusión de que todos ellos demostraron tencr participación inflamatoria glomerular, correspondiendo, por 10 tanto, a síndromes nefrósicos presentados durante el curso de glomérulo-nefritis.

Zarzar relata 12 casos observados en el Hospital Manuei Arriarán, entre los cuales sólo dos podrian considerarse nefrosis genuinas desde el punto de vista clínico, a saber: 
1) Niño de 5 años, cominilzo insidioso, anasarea, aibuminuria hasta 28 zre. bifoprotínemia de $45 \mathrm{grs.,} \mathrm{rusciente} \mathrm{bijo,} \mathrm{colestetinemia} \mathrm{de} 3.75 \mathrm{gts.}$ ausencia constante de bipertensión y hamaturias. Se desconoce su evolución desfués de 90 días de hospllalización. habiendo salido sin mejorarse, a pesar de los mulitiples teatamientos tealizados.

1) 6 añot. Edema generatizado, albuminutia hasta 48 gxs. hipoprorcinemia de $50 \mathrm{gts}$. Colestrrintmia de $3.55 \mathrm{~g}: \mathrm{s}$. Ausencia de húpertensión. Hématuria cscasa y aislada. Funcionalisno renz! normal. Se desconoce tambiér su colución posterior, por haberlo retirado ia familia $y$ ausentarse de la ciudad.

El resto de los casos ofrecen serias dudas respecto a la ausencia de compromiso glomerular, pues, o bicn presentaron hipertensión o hematurias de cierta consideración, en algunas ocasiones.

Fosteriormente hemos analizado 21 casos de posible nefrosis genuina. Cuacto observaciones, en todo semejantes a las anteriores, no permiten asegurar su existencia, por no conocer su evolución posterior. En 3 casos, cuya evolución clínica coincidia perfectamente con dicho diagnóstico, y que llegaron a término fatal. la autopsia reveló lesiones glomerulares de tipo inflamatorio. Los restantes carecen de un estudio completo para aceptar ese diagnóstico con seguridad.

Sin embargo, podemos presentar 2 observaciones, en que tanto la clínica como la anatomía patológica nos permiten. hasta donde podemos asegurarlo, con los medios a nuestro alcance, calificarlos como nefrosis genuina.

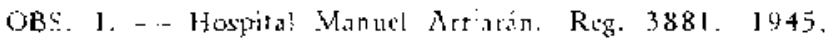

A. A. - 5 años de edad.

Antecedentes bereditarios. - Nagutivos patz tubercujosis y sililis.

Antecedentos personales. - Coqueillibe a los 2 años. Varicela a ios 4 meses. No acusa oteas afeccinics.

Enformedad actual. ... Después de un resfrio, una semana attás presenta edema, primero en la cars. el cusi pronto se genuraliza. Orinas escasas, de color amarillo. No acușa zafalea. Uno que otro vómito. Buen apetito y sed intensa. Ingresa con anasatca. Palidiz. Facics vuituosa. Tonos cardiacos bien timbrados. Pulso: 100 . Temperatura: 36, Presión: 90/75. Auscaltación pulmonar: disminución de! murmullo en las bases.

Examen de orina: Albúmina: $40 \mathrm{grs}$ o/oo. Tiscasos globulos blancos y nno que otro glóbulo rojo.

Uremia: $0,10 \mathrm{gr}$. $0 / 00$. Proteinas sanguineas: $48.5 \mathrm{ges} .0 / 00$,

Secreción faringea: Neumoroco $(++)$. Streptococo lemolitico $(-)$.

Fondo de ojos: Negativo. 
Evolución: La presión se mantienc siempre en limites normates. El todema tiende a disminuir. Al 5" dia de hospitalización aparece fitbre en relación con una placa de erisipeta del àdonica. Se hace una punción peritoneal. que da salida a un liquido rurbio. cuyo cultivo revela la ptésencia de neumococos. Se inicia tratamiento con penicilina. Fersiste la fiebre. Un nuevo examen de orina da: Albúmina: 10 grs. o/oo. La niña se agrava cada vez más y fallese at ton dia de su hospitalización.

Én la autopisia se comprueha lita piritonicis serofibrinosa generalizada neumocócica. Ieptomeningitis subagud. Chancro primatio tubetculoso del lébalo inferior del pulmén izquierdo con adenitis caseosa luberculosa.

Ambos riñones presentaban una tipoidosis generalizada a todos los túbulos de regular intensidad. dz preferencia en los contorncados. Hiperheria capilat. No se observan fesiones inflamatorias (Dr. J. Espinosa).

OBS. 2. -- Hospital Caḱvo Macksnad. --r Reg. $1123 / 47$.

N. L. - 2 años de idad.

Antcedentes hereditarios. - Sin importancia.

Ancecedentes personales. - Hace 10 meses difteria ratada zon $10.000 \mathrm{U}$. de antitoxina diftérica.

Al examen de ingreso sâ cuctintó mal estado general, con cdema discreto de los párpados y picrnas. Ascitis. Palidez intensa.

Diagnóstico de ingreso: Nefrosis. Anemia.

En el Servicio de recepción se cbicnen los siguientes sxínenes: Orina: aibúminas, 8,50 o/oo: ciíndros granulosos en regular cantidad. Glóbulós rojos ecasog. Uremia. $0.55 \% / 00$. Proteinemi. $41,6 \%$. Colestetinemia. $1.7 \% / 00$. Fondo dx ojos (-). Sexic tuberculiaica (.-). Hemograma: glób, tojos, 4.090.000; glób. blancos. 19,700; presión arterial, 100/70. Se piensa an nefrosis y sti ie indica régigren hiferprotxico. plasmolerapia y transfusiones. Posteriornente sc corsidera ja pasible existencia de lan gionérulonefritis eróniea y si regtringe la alimearatón $y$ por la crolución ligeramente febril y la leucocicosis, se instituỵ tataniento con perticilian a razón de 10,000 U. wada 3 horss. En visti de no rpreciarse mejoría y, por el ecntrario. un aumento enosiderable de su edema, se vuelve al régimetr hiperalbuminoidco y se instituye un tratrmiento con extracto tiroideo $(0.05$ gr. 2 reces al dis).

Conlinúa en malas condiciones: edema generalizado discreto. Gran ascitis con circulación colateral del abdomen. Edema del escroto. Presión, $100 / 60$. Orina: albuminas. 7 grs. Colesterinenía, 1,87. Proteinas, 43.8. Fondo de ojos normal. Uremia, 0,30. Conlinúa a régimen biperproteico. Se interrumpe el extrato tiro:deo por presentar signos de intolerancia. Plasmoterapia. Se hace una punción piritoneal, qué da salida a 2,300 grs. de líquido charo ransparente con caracier de transudado. Esta punción debe repetirse poio tiempo después. De acuerdo a los trabajos de Franconi. se le indica tratamiento con bicabonato de solio far os, en dosin erecientes de 4 a J2 grs. diarios. du- 
rante 10 días, sin ningún tesultudo. Posceriormente la uremia sobs a 0.60 gr. o/oo, presenta vómitos y diarrea con Reserva Alcalind de 63,3 vol. $\%$, Apsere fiebre y disnea $y$ el enfermo tallece.

Autopsia. - Exanen bistológica de los riñones: La sustancia cortical si aprecia engrosada. I.os giométulos en getneral se apreitan de aspecto nomal. Uno que otro muesta una discreta hislinización en su pediculo (vaso aference y clexente]. También se encuentra en algusos glomirulos un discreto edema en el interior de la capsula de Bowman.

Log tubulos muesiran su epittio muy altriado, ubicandose las lesiones en sus segmentos principales. El epitelio renal se ve en algunos puntos con escas zoloración nuclear $y$ el protoplasma binchado. de aspetto vacuolar, con io culal ol Jumen queda notablemente disminaido.

Ea otros puntos, el proteplasma se disgrega junto con el nácleo, viéndose en cl tumen masas filamentosas y finamene granulosas. Esta disgregación cslular llega a comprometis la totalidad del comono de! túbulo. qeedando úni. camentr de él la membrana basal, to que en conjunto da un aspecto de gra: des vacuolas con contenido grumosa, ubicado en las inmediaciones de los glomírulos. l.os segmentos intercalares te los cúbulos, frtegentan su epitelio más o nitzos en buenas condiciunes.

Conclusion: Intensa degeneración granular del epitelio renal, que llega basta su desintegración. Nefrosis.

Este último caso es $d_{i}$ difícil interpretación. En primer lugar. hubo ciertas dudas sobre si se trataba de una giomérulonefritis crónica, seguramente por la presencia de una ligera hematuria en exámenes aislados de orina y una relativa hipertensión (100/70): también existió el hecho de una uremia alta. en vísperas de su fallecimiento y manifestaciones probables de insuficiencia renal. En cambio, la sintomatología en genetal eta caracteristica de una nefrosis y su evolución cortespondió a tal afección. Sin embargo, la autopsia, si bien demostró la indemnidad de los glomérulos, salvo la presencia de discreta hialinización de algunos de ellos, reveló extensas e intencas lesiones degenerativas de los túbulos, pero sin las características de las lipoidesis renal. Es decir, una nefrosis del tipo de las descritas por Fahr como degeneracio$n$ as guturales hialinas a que hemos hecho mención al hablar de las nefrosis agudas. Probablemente. una etapa del proceso, previa a la degeneración grasa, que sería la característica de las formas más avanzadas.

Tal vez podriames comparar este caso con uno citado por Fishberg, del servicio del Dr. Herman Schwarz. en el cual no hubo hipercolesterinemia durante la vida y en cuya autopsia no se encontró tampoco presencia dz lipoides. En 
realidad, en nuestro caso, la cifra máxima de colesterol etr sangre alcanzó sólo a $1.87 \%$ \% puede decirse muy próxima de la normal. Tampoco se encontró presencia de cristales birrefringentes en la orina. Caso verdaderamente excepcional a juzgar de lo qu: dice el citado autor (Fishberg, pág. 367).

\section{Sindroma nefrósico.}

Como ya lo hemos señalado, si la nefrosis genuina es excepcional en nuestro medio. el sindreme nefrósico es de observación relativamente frecuente. ya sea como manifestación inicial de una glomérulo-nefritis o bien desarrollado durante el curso de ellas.

En el considerable número de glomérulo-nefritis que hemos tenido oportunidad de observar y que alcanza a más de 500 an estos últimos años, hemos encontrado una cifra aprcximada de $15.9 \%$ de formas. ya sea de evolución crónica o subcrónica y entre ellas, más o menos la mitad, o sea, un $7 \%$, ha presentado la sintonatología clásica, qu teaduce una participación nefrósica más o menos acentuada. En algunos casos el cuadro reveló iales caracteres de similitud. que s: hizo sumamente difícil discriminar si se trataba de una forma de nefrcsis genuina o de una nefritis-nefrósica.

Para ser breves relatamos a continuación sólo 2 casos de csta especie:

OBS. $7771 / 1945$

A. Z..... Edad: 4 años y 3 meses.

Antecedentes heredisarios y firsonales. -. Sin importancia.

Enfermedad actual. - .. Se inicia 15 dias atrás con edema de la cara, glee despues se generaliza. Orina escase y osebta.

A su ingreso ce compruebs anasatia $y$ l. existencia de una neumonia derébal. Presión, $7 / t y$ de pués $11 / 7$. Al 4 " dic de sul hospitalifación aparece

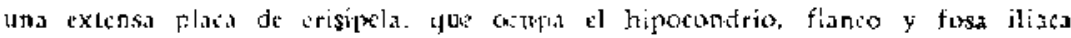
derecha basta al bipogastrio. So trata con sulfa y penicilina, mejorando tanto la neumonia como Ia erisipela. Propeinemia. 28.5 grs. o/co. Colesterinemia. 3.12. Albaminusia de mas di 15 grs. Hematuria y ciliadruria leves en ji: gunos eximenes. Urimis. 0.20. Sedimeotacion. 1 to. Secrecion faringea (-). Otorrino (---), Fondo de ojos (---), Con reposo, réginen declorurado, a base the lecte y de albumimas progresivamente aumenradas $y$ viromints, al $1^{\circ}$ dia disbace 7.500 grs. de edema. La albuminuria persistió alta hasta of dia 25 . I.a presión se notmalizó a los 22 dius de su ingteso. Sa proteinemia sulió a 48 grs. o/oo y la sedimentación bajo a $50 \mathrm{~mm}$. Las pruebas funcionales renaltes cran notmjales cuando fui dado de alla a los 37 dias de hospitalización. 
Reingresa un mes y medio después por presencar nuevamente edema grneralizado, anssarca. Se incicia as una nueva bospiralización, que duta esta vez más de un āio, durance la cual presenta edema constante de grado variable: abumiautia persistente que, en algunss ocosiones alcauza hasta 60 grs.; hematuria y cilindturia escasa, de vez en cuando. La presion arterial llega, en algunas otasiones, hasta 120, pero como rérmino medio se estabiliza en 90/60. La diufesis ticne grandes fluctuaciones, con conienlraciones altas y variables. Hifiecolesterinemia e hipoproteinemia constantes. Los numerosos bemogramas practirados revelan anemia hipoctoma. En 4 acasiones presentó euadros erisipeloides y procesos pulmonares agudos, que mejotaton con tratamiento de sulia $y$ penicilina. Experimenta remisiones del cuadro edematoso de corta duración, recayendo rápidamente. Se le scmete a difersos tratamientos a base de ragimenes sin sal, lácico puro, hambre y sed en alguna ocssión, preferentemente régimin hiperproteico, no obteniéodose ninguna mejoria duradera. Se usó también extracto tiroideo, que hubo de suspenderse en dos ocasiones por manifistar sintomas de intolerancia. Durante 58 díag se le ha tratado con este preparado en dosis progresivamente ascenderite. Se usó lambién plasmaterapiz en tosis de $200 \mathrm{~g}$ ts. diarios, durante 44 dias.

A los 285 dias de su hospitalización, en vista de la peristencia de la albuminoría y de los edemas más o nenos atentuados, se le instituye un régimen de tipo acidificante $(\mathrm{Sch} r \mathrm{~m})$. sin darle las grandes cantidades de líquidos gue se acorsejan para el adulto.

El régimen instiruío fué el siguiente: Desayuno $y$ onces: 225 grs. lo Fithe, $30 \mathrm{grs}$. de pan ton $15 \mathrm{grs}$ de mantequilla. Almuerzo $y$ comida: caldo con arroz o fideos, $100 \mathrm{grs}$ de carne, $100 \mathrm{grs}$. de verdura con $30 \mathrm{grs}$. de accitc. 50 grs. de par con 15 grs. de mantequilla. Ademas, se le suministraban 30 gotas de ácido clorbidrico al $10 \%$. 3 vetes al dia. Y 1 litro de una solución de glucosa al $50 \% / 00$ diario.

Con gran sorpresa nupstrā. se inició rápidamente una mejoria verdaderamente espectacular. Aumentó la diuresis hasta 1.400 grs. deshizo en 5 días $1,500 \mathrm{grs}$. de edema y éstos no volvizton más a reaparecer. Desapareció completanente la albuminuria (controlada en 7 exámcoes posteriores). Addis normal. La colesterinemia. que era de 3,34 antes de instituir el teatamiento, bajó cri menos de un mes a 1.90. La edimentación, que fué anta durante toda sul crformedad. comenzó a bajar a los 25 dias de tratamiento y permaneció normal basta el alra. Se inanturo el régimen especial durante 45 dias; postcriotmente se le mancuvo, por precaución, a régimen normal sin sal durante 10 dias $y$ durante cerca de un mes se nbsersó su tolerancia a1 tégimen normal, suspen. diéndose el roposo en anma. sir comptsbar ninguna alteración ni s su estado general ri en sus diversos exímencs de control. Las pruebas funcionales tenales practicadas antes del alta. eran no:males.

Autt no vuclve a control, de tal manera que ignoramos su estado 3ctuzl. 
En resumen, se trata de un niño que ha presentado un cuadto nefrítico-nefrósico de gran intensidad y prolongada evolución $y$ que ha curado $\mathrm{c} n$ forma, al parecer, completa, después de un régimen de tipo acidificante. Nos parece indudable su compromiso glomerular demostrado por la presencia de himaturia y de hipertensión; sin embargo, la evolución clínica es de una nefrosis genuina, de igual modo que su terminación espectacularmente favorable.

Otro caso que creemos de interés citar, sobre todo por su larga evolución y su mejoría también verdaderamente inesperada, es el siguiente:

O. B. - 6 años. Ingresa en eneto de 1938 a nuestro Servicio del Haspital Manuel hriatán.

Antecedentes hereditarios. - Sin importancia.

Ancesedentes personales. - Coquelucbr a fos 3 años. Piodermitis hace 2 años. Nuevo brote de prodeknitis haje un mis. Amigdalas sadas.

Enfermedad actual. - Se inicia 22 dias antes de su ingreso conl edema generalizado. Acude a la consulta extetna, donde se compraba: albuminuria dk 7 grs, o/oo y uno que otro glóbulo rojo. A pesar de un régimen cstricto. empeora y se hospilaliza. A su ingreso se efcuentra anasarca. Diuresis de 140.40-150 cc. diarios. Examen de crina: albúmina, 5 grs. $\% / c o$. Glóbulos rojos, regular cantidad, sin cilindrurix, Utemia, $0.52 \% / 00$ Colesterinemia. 2.10 grs. o/ 00 . Proteinas sanguineas: 78.8 grs. $0 / 00$. Serinas, 42.7 y globulinas, 36.2. Indice proteico: 1.17. Las presiones durante los primeros dias son de: $108 / 76-110 / 72$.

Evolución, - Fermanece 456 dius hospitalizado. Estuco sometido a lo.s más diversos y varindos regimenes y traranientos. Se obtiene durante cortos periodos mejorias apreciables, pero poco despuér sê intensifica de nuevo è cuadro, apareciendo albuminurias hasta de 30 grs. o/oo. acompañíndose d: enorme edema $y$ ascilis intersa. qua obliga a practicar punciones repetidas. extrayéndose varios litros de líquido jreritonea!. La hipoproteinemia se hace manifiesta $y$ la colesterinamia alcanza cifras hasia de $7 \mathrm{grs}$. $/ 00$, En gencral, nos encontramos anta un cuadro d: gravedad desesperante. Sólo la presión arterial permanece normal, aun cuanub en algunas ocasiones señala una máxima de 120 con minima de 80 (Baumanómetro). Después de varios meses de estada. habiendo fracasado toda clare de terapéuricas. estando el niño con enorme edema generalizado. albuminuria de $32 \mathrm{grs}$ o $/ 00$, gran ascitis, que obliga a practicar una nueva punción, qua da ralida 31,200 grs. de líquido. observamos que pocos dias nás tardi el niño inicia una sorprendente mejoría, regrzsando rápidamente todos sus sintomas. Deshace $7.500 \mathrm{grs}$, de edi. ma. la albuminuria desciende a 0,10-0.15 gr. o/oo. Uremia normal. Las pruebas funcionales del riñón. que en una ocasión habian revelado una Cons- 
tante fe Ambard de 0.13 , dan ahora zan cifra de 0.06 y las de concentracien y dilución dan tambien resultados nomales. Un primer control realizado 2 meses después de su alta, da rciultados completamente normales.

Controlado 2 años mís tarde da los resultados siguientes: Concentración: 1026. Dilución a 1,000. K. Ambard: 0,06. Uremia: 0.30. Addis (-), El niño se encuentra en condicionis, a lodo patecer, rormales.

Casc. como se ve, de dificil apreciación, pues su comienzo corresponde al de las mucioas formas de glomérulo-nefritis difusas, de etiología cutánea, que nos ha correspondido observar (hematuria, uremia alta), sin embargo, la participación nefrósica ha sido, sin duda, el carácter dominante y la mejoría tan inesperada en esté caso, también parece más propia de una nefrosis que de una glomérulo-nefritis subcrónica.

Podría también servir este caso para demostrar la teoría de que en el comienzo de toda nefrosis existe primero una glcmérulo-nefritis, que muchas veces pasaria inadvertida y aún curaría, bastando esa alteración glomerular para desencadenar el proceso tubular degenerativo.

Mucha mayor extensión podría tener este trabajo si publicáramos todas y cada una de las numerosas observaciones de tipo semejante a las anterioles que están en nuestro archivo: pero creemos que es innecisario y que basta con lo cxpuesto para terminar sometiendo a la consideración del Primer Congreso Panamicano de Pediatría las conclusiones si. guientes, deducidas de la revisión de cerca de un millat de observaciones clínicas da nefropatías de la infancia que han pasado por los servicios hospitalarios de Santiago de Chile desde el año 1930 hasta la fecha actual.

1. La nefrosis genuina (Volhard y Fahr), si bien no podemos negar su existencia como tal, es extraordinariamente rara en Chile.

2. Los casos publicados en ruestra literatura ofrecen serias dudas respicto a la exactitud de su diagnóstico.

3. El sindroma nefrótico (Nephrotic - Nephritis). acompañante de una glomérulo-nefritis, se observa, en cambio, con relativa frecuencia en nuestras nefropatías.

4. Hacemos risaltar la notable frecuencia de la glo mérulo-néfritis en nuestro pais, principalmente en el medio sccial pobre. influida segurament: por factores de orden infeccioso cutáneo y carenciales. 


\section{Summary.}

The author presents this report to the first Pan-American Congress of Pediatrics, in which, after making a revision in the general concepts of nephrosis in childhood, he explains the experimentation carricd on in Chile through an analysis of cases studied in the principal centers of child care in the capital city.

For this paper he has divided nephrosis into two types: the acute and the chronic. The former appears in the progress of some febrile affections, and while though of an evolution generally mild. may, in some caszs, manifest itself with grave symtoms and may even cause death through nechrosis of the parenchyma. Quite frequently the discovery of these cases was the result of autopsy.

The chronic forms of this malady deserve especial consideration, and the author concerns himself principally with the much-discussed problem of whether or not, according to the concepts of Volhard and Fahr, a genuine lipoid form exists. After a revision of the works of different investigators and in agreement with his own experimentation, he synthesizes the present stage of the discussion in the following form:

1) While the genuine lipoid nephrosis is extremely rarc, even in its favorite age, childhood, its existence still cannot be denied, for several authors of distinction have cited numerous cases in which the illness present:d itself witb all its symptomatic gallantry and with a total absence, even histolcgical, of glomerular alterations of an inflamatory nature.

2) Chrcnic nephrosis would probably not be an original disturbance of the metabolism of the albumin and lipcides, but rather its pathogenicity would reside in an original alteration of the renal parenchyma: and even when it is net plain!y demonstrated what the nature of this primary process is, it is generally believed that the initial lesion would produce itself in the glomerulous which would be allowed to pass albumin necessary to the organism, and more over the nutrition of the tubuli wculd be disturbed, and that both alterations would bring as a consequence the development of the degenerative processes of the tubuli.

This glomerular alteration would probably be of a mild and transitory character, and in many instances it would not be detected by the usual means of investigation; giving 
way to the so-called genuine lipoid nephrosis; but in other instances it would clothe the characteristics more or less apparent with a glomerular nephritis, and the production of the nephrosis syndrome, which with relative frequency is observed in the course of wide-spread glomerulonephritis in a chronic or subchronic form, would then be clear.

It is indubitable that, in some cases at least. this is produced in persons in whom exists a disturbance probably of the functional metabolism or in the constitutional character on a pathologically endocrine basis.

The author analyzes briefly the etiology. the patholog:cal anatcmy, the treatment, and the prognosis of he affection. annotating the present condition of already known facts.

Immediately afterwards the writer describes the experimentation conducted in his country by divers Chil an investigators and adding to it his own experience thrcugh several hundred cases of nephropatias observed in childheod during th: last ten years. he has succeeded in compiling about fifty cases of presumed lipoid nephresis, of which only two or three can be considered as such. from a clinical as well as anatomepathological viewpoint of this diagnosis. He then give; a report of these pertinent observations, one of which corresponds with cxactitude to a purely lipoid form. while th: other represent quite rare histopathological particularities.

Referring to the nephrotic syndrome of these many casis. he presents only two rather characteristic observations. pointing out the great difficulty of arriving at a precise diagnosis.

$\mathrm{He}_{2}$ terminates this summary by submitting to th: consideration of this Congress the following conclusions:

1) The genuine lipoid nephrosis (Vothard and Fahr), while we cannot deny its existence as such is extremely rare in Chile.

2) The cases published in our literature prisent serious dcubts in regard to an exact diagnosis.

$3 j$ The nephrotic syndrome accompanied by a glomerular nephritis is observed with relative frequency in our cases of nephropatias.

4) It is evident that the notable frequency of glom:rulonephritis in our country, principaliy among the poor:r classes, is due principally to skin affections and the lack of proper food. 


\section{Bibliografía.}

Al.DRlCII. - Brineman Practice of Pediatrics. Vol, III. 25-20.

BAEZA GOÑl, A. - L.a Gomérutonefritis en la Infancia. Editorial Zig-Zag. Santiago de Chile. 1942 .

BAEZA GOSI. A.: ERNESTINA FEÑA y A. OYARZUN -- Nef dea. Rev. Chilena de Pedialtia. 1930. Pig. 189.

BAUZA $y$ HERNANDEZ. - El Sindroma Kíctrósico en la Iníncia. Archi wes del Hospital Robetto del Río. Marzo de 1945.

CEClL. - - Textbook of Medicine.

DE SANCTIS y SUL.LIVAN. - Nephrosis. Jnurnal of Pediatrics. 1947.

I'ISHBERG. - Hyprtension and Nephritis.

GARRAHAN. - Meticina Infantis.

GRIFFITH MITCHELL. - Textbook of Pediarics.

HADIIELD and CARROD. - Rcents Advanes in Pathology. 1943

HEYMAXN and STRRTZMAY. - Nepbrosis. Jousnal of Fediatrics. Jebr $19+6$.

HOL.T and MC INTOSH. - Diseases of Infatucy and Childhond.

JIMENГZ DIAZ. - lecsiones di Patologia Medica. Tomo It, 4' Edic. I945

I.I.NEWEH. - - Pacogenia y tratamicnto d.e l, Nefrosis en la Infancia. Mo

nasr. F. Kinderlueil. 74, 3 y 6

'TRUETA $y$ colabotadores. - Rasal Patbology in the light of rezent neurcrisculat Studies. Lancet. Ag 1946.

VARELA M., ENRIQUE. - Nefropaias. 89 lid. 1941.

WIEDERHOLD, A. - la Nefrosis en la Infancia. Revista Chilena de Pedintria, 1939. Pig. 465.

ZARZAR. - Siudroma Nérósico an la Infanria. Rerista Chilena de Pediatrin 1946. Píg. 195. 